\title{
Kernos
}

Revue internationale et pluridisciplinaire de religion grecque antique

$2 \mid 1989$

Varia

\section{Conception et manifestations du sacré dans l'Hymne homérique à Aphrodite}

\section{Vinciane Pirenne-Delforge}

\section{OpenEdition \\ Journals}

\section{Édition électronique}

URL : http://journals.openedition.org/kernos/248

DOI : $10.4000 /$ kernos. 248

ISSN : 2034-7871

\section{Éditeur}

Centre international d'étude de la religion grecque antique

\section{Édition imprimée}

Date de publication : 1 janvier 1989

Pagination : 187-197

ISSN : 0776-3824

\section{Référence électronique}

Vinciane Pirenne-Delforge, « Conception et manifestations du sacré dans l'Hymne homérique à Aphrodite », Kernos [En ligne], 2 | 1989, mis en ligne le 02 mars 2011, consulté le 20 avril 2019. URL http://journals.openedition.org/kernos/248; DOI : 10.4000/kernos.248 
Kernos, 2 (1989), p. 187-197.

\section{CONCEPTION ET MANIFESTATIONS DU SACRÉ DANS L'HYMNE HOMÉRIQUE A APHRODITE*}

Quel que soit le point de vue adopté dans l'étude du sacré, la notion évoque immanquablement l'idée d'une relation, d'une communication entre deux pôles, le divin et l'humain ${ }^{1}$ et l'on attend d'une religion qu'elle réglemente le passage d'un temps déterminé à une infinité éternelle où l'homme peut courir un danger ${ }^{2}$.

Chaque groupe humain possède ses règles propres, codifiées ou non, visant à harmoniser la coexistence du sacré et du profane et à instaurer un équilibre viable entre une existence à la mesure humaine et un ordre divin irréductible à cette mesure même.

La Grèce a raconté, par la voix de certains de ses poètes, les relations entre les hommes et leurs dieux. Et ces différents récits, dans la mesure où l'on peut les rapprocher et les comparer, offrent un panorama cohérent des manières d'appréhender le sacré en Grèce, et de vivre avec lui. Les deux œuvres majeures d'Hésiode, la Théogonie et Les Travaux et les Jours, ainsi que certains hymnes homériques, tout aussi anciens ${ }^{3}$, sont de ces textes-là. Ils disent la genèse du sacré, le pourquoi d'un type de relation défini entre les dieux et les hommes.

Le présent travail entend montrer que l'Hymne homérique à Aphrodite constitue un maillon important dans l'élaboration de ce modus vivendi qui concerne autant les dieux entre eux que leurs rapports avec les hommes ${ }^{4}$.

* Cet article est le texte remanié d'une communication présentée dans le cadre du XIIe Congrès de l'Association Guillaume Budé qui s'est tenu à Bordeaux en août 1988 et dont le thème était «Les écrivains et le sacré».

1 A. MoтTe, L'expression du sacré dans la religion grecque, in L'expression du sacré dans les grandes religions, III, Louvain-la-Neuve, 1986 (Homo Religiosus, 13), p. 112.

2 R. CAIlloIs, L'homme et le sacré, Paris, 1939 (Mythes et Religions), p. 1-5.

3 R. JANKo, Homer, Hesiod, and the Hymns, Cambridge, 1982 (Cambridge Classical Studies), p. 179-180.

4 Nous voulons dire ici notre dette à l'égard des travaux de Jean RUDHARDT qui, outre son importante thèse sur les Notions fondamentales de la pensée religieuse et actes constitutifs du culte dans la Grèce classique (Genève, Droz, 1958), a centré sa réflexion sur l'étude du mythe comme langage particulier en s'attachant notamment, pour illustrer son propos, à l'étude des grandes étapes de la mise en ordre du cosmos : Du mythe, de la religion grecque et de la compréhension d'autrui, Genève, Droz, 1981 (= tome 19 de la Revue européenne des Sciences sociales, Cahiers Vilfredo 


\section{Prolégomènes}

Chez Homère, Aphrodite est fille de Zeus et de Dionè ${ }^{5}$. Dans la Théogonie, elle est née du sexe d'Ouranos tranché par Cronos et jeté dans la mer ${ }^{6}$. Et, dès le début, nous dit Hésiode, son privilège, sa timè, sa moira, parmi les hommes et les dieux immortels, ce sont les conversations de jeunes filles, les sourires, les duperies, le plaisir suave, la tendresse, la douceur ${ }^{7}$. À quelques différences près, ces attributions se trouvaient déjà dans l'Iliade ${ }^{8}$. Mais l'écart entre les deux cuvres est de taille: Homère fait d'Aphrodite la fille de Zeus dans le cadre d'une famille soumise à l'autorité du père; Hésiode en fait la fille d'Ouranos, la sœur des Titans, la «tante» de Zeus, une déesse aux privilèges déterminés dès l'origine. Homère montre Aphrodite soumise à l'autorité de Zeus en un temps où les grands conflits cosmogoniques sont révolus et où les attributions respectives des dieux ont été déterminées sous la houlette du père divin. Hésiode, quant à lui, dépeint les épisodes tourmentés des origines du monde à une époque où les forces de l'hybris s'opposent aux desseins éternels de Zeus. Aphrodite appartient directement à cette époque et, avec elle, le cortège de ses privilèges 9 .

D'où surgit la question: dans quelles circonstances cette déesse ancestraJe toute-puissante a-t-elle dû obéissance à Zeus ? Une telle interrogation pourra paraître naïve, et pourtant l'Hymne à Aphrodite paraît bien y apporter une réponse ${ }^{10}$.

Vilfredo Pareto); Pandora: Hésiode et les femmes, in $M H, 43$ (1986), p. 231-246; Le rôle d'Éros et d'Aphrodite dans les cosmogonies grecques, Paris, P.U.F., 1986.

5 Iliade, $\mathrm{V}, 370-417$.

6 Théogonie, 188-206.

7 Ibid.

8 Iliade, XIV, 159-212. Il s'agit de l'épisode où Hésiode montre Zeus berné par son épouse. Cf. V. PIRENNE-DELFORge, La mere, la fille, l'amante et l'épouse. Les déesses grecques, Univ. de Liège, 1988 (Entretiens sur l'antiquité gréco-romaine), p. 2-4.

9 Il n'est pas question de nier l'antériorité historique d'Homère par rapport à Hésiode, mais de mettre en évidence l'antériorité "mythique» des faits que raconte Hésiode.

10 La bibliographie concernant l'Hymne est considérable. Ne seront ici mentionnés que les travaux desquels cette contribution est le plus redevable et qui reprennent les études antérieures. H.N. PORTER, Repetition in the Homeric Hymn to Aphrodite, in AJPh, 70 (1949), p. 249-272; J.C. KAMERBEEK, Remarques sur l'Hymne à Aphrodite, in Mnemosyne, 20 (1967), p. 385-395; F. JouAN, Thétis, Hestia et Athéna, in REG, 69 (1956), p. 290-302; L.H. LENZ, Der homerische Aphrodite Hymnus und die Aristie des Aineias in der Ilias, Bonn, Habelt, 1975 (Habelts Diss. Dr R. Klass. Phil., 19); Ch. SEgAL, The Homeric Hymn to Aphrodite: A Structuralist Approach, in CW, 67 (1974), p. 205-212; N. VAN DER BEN, De homerische Aphrodite-Hymne 1 : De Aeneas-passages in de Ilias, in Lampas, 13 (1980), p. 40-77 : cet article remarquable 


\section{L'Hymne homérique à Aphrodite. Rappel}

"Muse, dis-moi les travaux d'Aphrodite d'or». Dès le premier vers, l'auditeur, le lecteur est averti des desseins du poète : énoncer les ě $\rho \gamma \alpha$

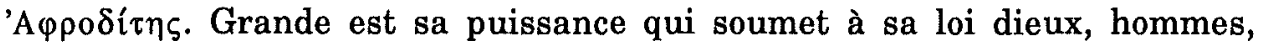
animaux ${ }^{11}$. Le fait même d'énoncer les exceptions dans l'application de ses pouvoirs - le cas d'Athéna, d'Artémis et d'Hestia - n'est qu'une manière de les exalter d'autant. Même Zeus ne peut échapper aux desseins d'Aphrodite qui le fait s'unir à des femmes mortelles, dans l'oubli momentané d'Héra, son épouse ${ }^{12}$. Mais Zeus entend arrêter la déesse dans son œuvre : il veut à son tour lui insuffler l'amour pour un mortel et l'empêcher ainsi, nous précise le texte, «de déclarer parmi tous les dieux avec un rire suave qu'elle a uni des dieux à des femmes mortelles, qui donnèrent aux Immortels des fils mortels - et aussi des déesses à des hommes mortels»13. En somme, Aphrodite a abusé de son pouvoir en s'ingéniant à unir mortels et immortels, et c'est dans cet exercice particulier de sa puissance que Zeus désire l'arrêter. Il lui met dans le cour l'amour pour Anchise «qui tient des dieux sa beauté» et elle entreprend de le séduire.

Au premier abord, la déesse veillera à ne pas apparaître dans le rayonnement de sa divinité. Dans son domaine parfumé de Chypre, les Charites la parent d'immortalité, mais c'est en simple mortelle qu'elle se présente devant Anchise afin qu'il n'ait pas peur en l'apercevant ( $\mu \eta^{\prime} \tau \alpha \rho \beta \eta ́ \sigma \varepsilon\llcorner\varepsilon v)$. De fait, le pâtre n'est pas saisi de crainte mais bien d'un étonnement admiratif ( $\theta \alpha v \mu \alpha i ́ v e v v)$ que suscitent l'éclat de la personne et celui de la parure. La beauté de la femme est telle qu'elle ne peut être que déesse ${ }^{14}$, et Anchise la

passe en revue les principales études sur l'Hymne afin d'éprouver la pertinence de la théorie d'une "famille des Énéades" qui aurait commandité la composition du poème; N. VAN DER BEN, De homerische Aphrodite-Hymne $2:$ Een interpretatie van het gedicht, in Lampas, 14 (1981), p. 67-107; P. SMITH, Nursling of Mortality. A Study of the Homeric Hymn to Aphrodite, Bern, Lang, 1981 (Stud. zur hlass. Philol., 3).

11 V. 1-5. Cf. Esch., fr. 44 Nauck $^{2}$; EuR., Hipp., 447-450.

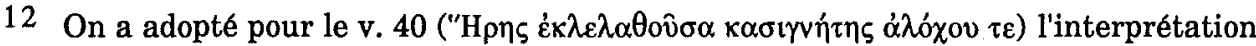
de J.C. KAMERBEEK, art. cit., p. 390, qui met davantage l'accent sur l'oubli de Zeus que sur sa tromperie (cf. HUMBERT, dans l'édition de l'Hymne dans la Coll. des Univ. de France, p. 152 : «à l'insu den).

13 V. 48-52. N. VAN DER BEN (II, p. 92-93) a bien montré qu'il s'agissait non pas d'une *humiliation» d'Aphrodite, mais bien d'une limitation de ses pouvoirs. Elle doit s'arrêter d'agir de la sorte, et pas seulement cesser d'en parler.

14 La beauté est un des critères essentiels de la reconnaissance du divin par l'homme car l'homme qui possède la beauté ne peut la tenir que des dieux. Ainsi, dans 
prie de lui accorder le bonheur. Suit alors le long discours de la déesse qui se défend d'en être une et reporte sur Hermès - qui l'aurait enlevée l'impression d'immortalité ressentie par Anchise. Et l'homme beau comme un dieu tombe amoureux de la déesse qui fait la mortelle. Convaincu maintenant d'avoir affaire à une femme, Anchise exprime sans embages son désir : rien ni personne ne l'empêchera de s'unir à elle immédiatement. Et le mortel de se coucher auprès d'Aphrodite sans le savoir vraiment. Le réveil sera rude. La déesse en majesté interpelle son amant endormi et celui-

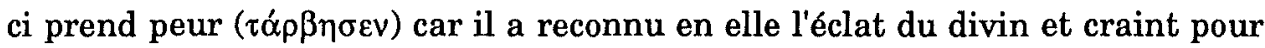
sa vie. Aphrodite le rassure : il ne doit pas éprouver de crainte. Il est aimé des dieux. C'est elle, la déesse, qui a failli et ressent une terrible angoisse. Elle explique ensuite que les hommes de la race d'Anchise ont souvent rejoint les immortels, mais elle lui signifie également, par les exemples qu'elle évoque, qu'il ne l'accompagnera pas dans la société olympienne : le blond Ganymède, enlevé par Zeus, restera à jamais un bel adolescent, Tithon, aimé d'Aurore, est un vieillard immortel qui radote au fond d'un palais divin. Aphrodite ne veut pas de cela pour Anchise qui restera un homme parmi les hommes et vieillira au milieu des siens. La déesse retournera elle aussi parmi ses pairs, mais la situation aura changé : son ingéniosité les pliait sous sa loi, ils la craignaient ( $\left.\alpha_{\alpha} \rho \beta \varepsilon \sigma \kappa o v\right)$, mais ce ne sera plus le cas désormais. Quant à l'enfant à naître, il sera pris en charge par les Nymphes de la montagne, celles qui ne sont ni mortelles, ni immortelles, avant d'être rendu à son père qui l'emmènera dans la cité de Troie. Et si un mortel demande qui est la mère d'Énée, Anchise devra répondre évasivement, sans nommer Aphrodite, sous peine d'être foudroyé par Zeus ${ }^{15}$.

\section{La place de l'épisode dans l'économie générale du cosmos}

Ce poème célèbre la puissance d'Aphrodite, mais il présente aussi, implicitement, les limites que Zeus va lui imposer. A défaut de la lui avoir entièrement accordée, le dieu va en régir les applications.

Les œuvres de la déesse font s'unir les dieux, les hommes et tous les êtres de la création en leur insufflant du désir. Aucune créature n'échappe à cette loi qui, pour être saine et équilibrée, doit unir le semblable : les dieux à leurs

l'Hymne, Anchise, Ganymède, Tithon et Énée sont pareils à des dieux en vertu de leur beauté.

15 On ne s'attadera pas ici sur l'interprétation de cette menace finale, d'autant moins compréhensible que, aux vers 192-195, la déesse a pleinement rassuré Anchise sur son sort. Peut-être faut-il y voir, comme N. VAN DER BEN (II, p. 96-99), une ironie du poète qui place dans la bouche d'Aphrodite une possible sanction de Zeus à l'égard de l'humain, alors que c'est elle-même que le dieu punit dans le récit ! 
pairs, les humains entre eux et les animaux en fonction de leur espèce. Or il existe un déséquilibre dans ce processus général. Bien après que les humains ont quitté la compagnie insouciante des dieux, mortels et immortels continuent épisodiquement de s'unir év $\varphi \iota \lambda c^{\prime} \tau \eta \tau \imath ~ \pi \circ \lambda v x \rho v ́ \sigma o v$ 'A $\varphi \rho \circ \delta i \tau \eta \varsigma^{16}$. Ce que les hommes ont perdu sur le plan alimentaire, certains d'entre eux semblent l'avoir conservé sur le plan affectif et sexuel. Et des déesses portent encore les enfants de mortels, tandis que des femmes humaines sont mères par l'entremise de dieux immortels.

Mais cette promiscuité doit cesser car elle va l'encontre du nouvel ordre voulu par Zeus. L'homme et le dieu, les deux pôles du sacré, doivent désormais tenir leur place dans la sphère qui leur est propre. Cette ultime séparation est racontée dans l'Hymne à Aphrodite qui en explique clairement les raisons. L'homme est mortel et connaît la funeste vieillesse; le dieu jouit d'une éternelle jeunesse et a en horreur la dégradation corporelle qui conduit au royaume d'Hadès ${ }^{17}$. L'union physique du divin et de l'humain met donc en contact direct mortalité et immortalité. L'inconciliable, en somme. Et les protagonistes le savent : Anchise craint pour sa vie ${ }^{18}$ et Aphrodite est prise d'une "terrible angoisse» 19 : sa relation avec Anchise est nécessairement éphémère et la vieillesse lui est d'autant plus insupportable que ses épy $\alpha$ sont par excellence l'apanage de la jeunesse ${ }^{20}$. De plus, dans le cas présent et en fonction du plan de Zeus, elle voit ses pouvoirs délimités à jamais.

Ainsi Zeus, sans modifier la nature originelle de la timè d'Aphrodite, l'a en quelque sorte canalisée en empêchant définitivement que l'ordre du monde mis en œuvre tout au long de la théogonie ne soit perturbé par la mètis

16 Sur la séparation des dieux et des hommes lors de la crise de Mécôné, voir J. RUDHARDT, Les mythes grecs relatifs à l'instauration du sacrifice : les rôles corrélatifs de Prométhée et de son fils Deucalion, in op. cit., p. 214-217 et Le mythe hésiodique des races et celui de Prométhée. Recherche des structures et des significations, in op. cit., p. 272-277.

17 Le vers 246 de l'hymne est très clair de ce point de vue. Cf. aussi J. RudHARDT, A propos de l'Hymne homérique à Déméter, in op. cit., p. 235-236. - Un fragment de Pindare, cité à plusieurs reprises par Plutarque (Moralia, 167e et 763c) définit les

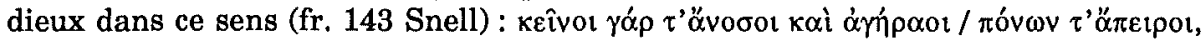

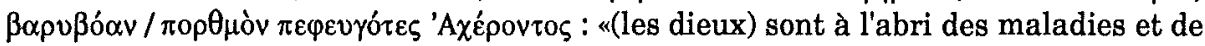
la vieillesse et de l'effort, et des cris sourds de ceux qui passent l'Achéron» (d'après R. Flacelière).

18 N. VAN DER BEN, art. cit., II, p. 80.

19 aivòv öxos : v. 198-199.

20 Cf. Hymne homérique à Déméter, v. 101-102; Platon, Banquet, 194b : l'amour s'attache à la jeunesse et fuit la vieillesse. Voir tout particulièrement à ce sujet : EUR.,

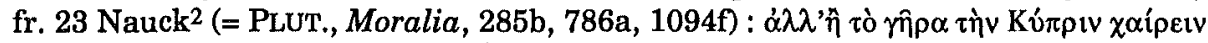

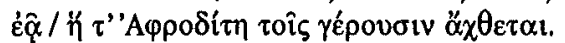


d'une déesse ${ }^{21}$. Il est inconcevable que la société divine continue de craindre Aphrodite $^{22}$ qui devient pleinement fille de Zeus, comme le montre clairement l'étude des épithètes qu'elle porte tout au long de l'hymne. Пo

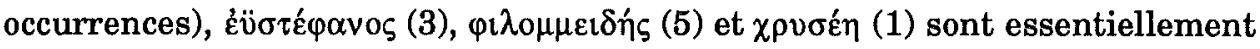
utilisées dans des contextes amoureux, lors de l'affirmation de sa puissance sur tous les êtres et lors de ses apprêts à Chypre. Quant à l'appellation $\Delta$ iò

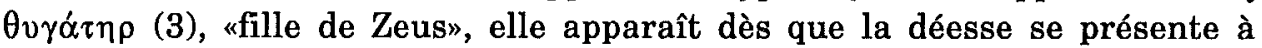
Anchise. En somme, dès que le plan de Zeus s'est irrémédiablement mis en route (v. 81). Aphrodite est encore fille de Zeus lorsqu'elle ment à Anchise (v. 107) et enfin quand elle s'adresse à lui en tant que déesse (v. 191) ${ }^{23}$.

L'Hymne homérique à Aphrodite explique également pourquoi le temps des héros est révolu. Les demi-dieux ont disparu et les hommes se retrouvent entre semblables. Cette situation transparaît nettement dans la prière qu'Anchise adresse à son interlocutrice, croyant se trouver en présence d'une déesse : il veut vivre reconnu parmi les siens, être père et connaître le bonheur, dans la prospérité jusqu'à la vieillesse ${ }^{24}$. On est loin de l'idéal de vie du héros. Et si, après s'être uni à la déesse, il désire un moment l'accompagner parmi les immortels - la demande est rendue implicite par la réponse d'Aphrodite qui raconte ce qu'il est advenu de Ganymède et de Tithon ${ }^{25}$-, c'est qu'il redoute de vivre affaibli ${ }^{26}$ parmi les siens et de ne pas «voir fleurir sa vie» (v. 189). Il craint donc de ne pouvoir réaliser ses aspirations d'humain mortel.

21 V. 249. - Sur la mètis particulière d'Aphrodite, cf. M. DETIENNE-J.-P. VERnANT, Les ruses de l'intelligence. La mètis des Grecs, Paris, 1974 (Nouvelle bibliothèque scientifique), p. 267-268.

22 V. 249-251 : le verbe utilisé pour signifier la crainte de dieux, $\tau \alpha \rho \beta \hat{k} \omega$, est celui qui désigne la peur d'Anchise (v. 83, 182).

23 Voir les remarques intéressantes de D. DicKMANN BoEDEKER, Aphrodite's Entry into Greek Epic, Leiden, Brill, 1974 (Mnemosyne Suppl. 32), p. 20-42.

24 V. 102-106. Cf. P. SMITH, op. cit., p. 46-49.

25 P. SMITH, op. cit., p. 71-86; N. VAN DER BEN, art. cit., I, p. 79.


«castration" de type asiatique que Cybèle inflige à ses amants (par ex., J.H. Rose, Anchises and Aphrodite, in CQ, 18 [1924], p. 11-16). Cf. P. SMITH, op. cit., p. 8-9. Anna Giacomelli (Aphrodite and After, in Phœnix, 34 [1980], p. 1-19) a étudié très précisément le sens de cette "perte de $\mu \varepsilon v o ́$ s" que sous-entend l'épithète. Et la question qui est posée n'est plus de quoi Anchise a peur, mais bien pourquoi il a peur que ce rapport sexuel ait tari son alimentation de $\alpha \mu \varepsilon v o ́ s »($ p. 18) ? Simplement parce que la sphère des mortels et celle des immortels n'ont aucune commune mesure. N. VAN DER BEN (II, p. 80) critique, sans justification vraiment convaincante, cette interprétation qui, pourtant, ne nous semble pas être en contradiction avec sa propre analyse. 
Au contact de l'immortel, l'humain risque d'altérer l'intégrité même de sa nature mortelle, mais il semble bien que cet "affaiblissement» de l'homme résulte d'une volonté délibérée du dieu plutôt que de l'acte sexuel en lui-même 27 . Et Aphrodite de le rassurer : lui, il n'a rien à craindre. Le châtiment sera pour elle seule ${ }^{28}$. On peut même affirmer que l'humain sort grandi de l'aventure. Lui n'a rien perdu de son humanité, alors que la déesse a déchu.

Pour la première et la dernière fois, la déesse de l'amour est descendue parmi les hommes ${ }^{29}$, dans ce lieu intermédiaire entre ciel et terre qu'est l'Ida aux mille sources ${ }^{30}$. Et l'enfant à naître, dernier représentant des hemi-theoi, connaîtra en premier lieu un monde intermédiaire : celui des Nymphes de la montagne «que l'on ne compte ni parmi les êtres mortels, ni parmi les immortels", nous dit le poète ${ }^{31}$. Elles meurent pourtant, mais, au regard de la vie humaine, la leur relève presque de l'immortalité. Pas au point, cependant, d'appartenir à la société des dieux. Ce caractère particulier des Nymphes, qu'Homère situe parmi les immortels ${ }^{32}$, s'inscrit remarquablement dans l'économie générale du poème car leur existence même accentue encore l'altérité entre les deux pôles de la création, tout en comblant un vide dans la hiérarchie des êtres ${ }^{33}$.

Aphrodite a donné un enfant à un mortel, mais sa maternité met fin à la procréation des dieux, lesquels n'étaient plus féconds ${ }^{34}$ désormais hormis dans leurs relations avec des humains. Le monde des dieux est défini dans le nombre de ses représentants. La relation sexuelle, désormais normalisée,

27 Cf. Odyssée, X, 287-363 : Ulysse fait jurer à Circé qu'elle ne lui fera aucun mal en lui offrant de partager sa couche. Elle jure le grand serment des dieux et le héros sort indemne du lit de la déesse.

28 V. 247-254.

29 A la fin de ce qui nous reste de la Théogonie, Hésiode chante «les immortelles entrées dans le lit d'hommes mortels, qui leur ont enfanté des fils pareils aux dieux» (v. 9651020). Aphrodite y est mentionnée deux fois : en tant que mère d'Énée (v. 1008-1010), mais également comme ravisseuse de Phaéton, fils de Céphale et d'Aurore. On s'aperçoit bien vite que cet épisode n'est rapporté ici que dans la mesure où Phaéton est fils d'une déesse et d'un mortel, et pas nécessairement amant d'Aphrodite. Il est

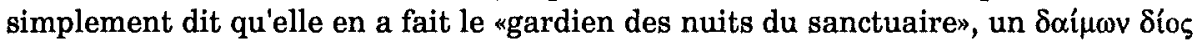
(v. 989-991).

30 T. ORY, L'animal et le végétal dans l'Hymne homérique à Aphrodite, in LEC, 52 (1984), p. 252.

31 V. 259.

32 lliade, $\mathrm{XX}, 4-12$.

33 Cf. N. VAN DER BEN, art. cit., II, p. 94; P. SMIIH, op. cit., p. 93.

34 J. RUDHARDT, Le rôle d'Éros et d'Aphrodite, p. 37. 
reste l'œuvre d'Aphrodite qui donne continûment aux hommes le pouvoir d'enfanter. Et c'est peut-être le sens qu'il faut donner, d'une manière générale, aux mots rassurants d'Aphrodite : ne crains rien, tu verras fleurir ta vie et tous les hommes avec toi ${ }^{35}$. La vieillesse, tant détestée des dieux, les hommes peuvent l'affronter après avoir tiré d'eux-mêmes une nouvelle jeunesse. L'homme est toujours destiné à la mort - ce thème est récurrent, voire obsédant tout au long du poème $e^{36}$ - mais, en donnant la vie, il se survit à lui-même.

Il semble donc que la figure de l'enfant soit plus centrale qu'il n'y paraît de prime abord. C'est lui le principal point de contact entre le mortel et l'immortel; il est intermédiaire, tout comme les nourrices semi-divines qui l'élèvent. Même s'il porte en lui sa mort à venir, il est le signe de cette belle jeunesse tant aimée des dieux, qui refleurit à chaque génération.

L'union d'Aphrodite et d'Anchise relève bien du sacré en ce qu'elle consacre mythiquement une communication spécifique entre le divin et l'humain. Elle signifie également le terme d'une modalité particulière de contact. Et la conséquence en est fondamentale : l'homme et la femme se retrouvent face à face, forts de ce pouvoir contre la mort qu'est la procréation. Il est évident que les ép $\gamma \alpha$ 'A le rappelle le début du poème. Mais cette union est désormais une des seules voies qui permette à l'homme d'atteindre une forme d'immortalité. C'est au creux de la relation charnelle et dans la venue d'un enfant que l'humain poursuivra l'œuvre divine d'ordonnancement du $\operatorname{cosmos}^{37}$.

\section{Aphrodite et le sacré. Spécificité de la déesse}

La spécificité d'Aphrodite apparaît a contrario dans la présentation de celles qu'elle ne peut ni persuader ni séduire ${ }^{38}$. Athéna, fille de Zeus, aime la guerre, les travaux d'Arès. C'est elle aussi qui enseigne leur art aux artisans et les nobles travaux aux tendres vierges. Artémis aux flèches d'or, pour sa part, aime le massacre des fauves sur la montagne, la musique, les chants, les danses, les bois et la cité des Justes. Ces deux filles de Zeus,

35 V. 196-197.

36 Le poète fait alterner le vocabulaire de la mortalité et celui de l'immortalité : $\theta$ cós ou

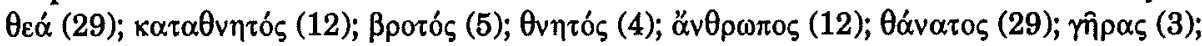

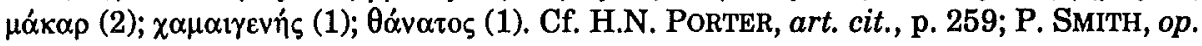
cit., passim.

37 En effet, les dieux ont besoin des hommes : cf. H. h. Déméter, 310-312; Platon, Banquet, 190c.

38 V. 7-35. Cf. F. JOUAN, art. cit. 
placées sous l'autorité paternelle dès l'origine, ont une prédilection pour les œuvres de mort que sont la guerre et la chasse. Athéna remplit également une fonction didactique: elle enseigne les arts aux humains ${ }^{39}$. Artémis patronne les activités typiques des vierges aristocratiques que sont les chœurs ${ }^{40}$. Quant à Hestia, elle a juré de rester vierge. Elle n'est pas fille de Zeus, mais bien de Cronos. Cependant, c'est Zeus qui a défini son privilège : honorée par les mortels, elle est le foyer du sacrifice au cœur de la maison.

Ainsi, non seulement ces déesses sont vierges, mais elles patronnent diverses formes de mise à mort ${ }^{41}$ et leurs relations aux humains diffèrent totalement de celles qu'Aphrodite entretient avec eux. En effet, la déesse est, au départ, indépendante de Zeus; elle préside à la vie qui naît et s'épanouit par son entremise. Son intervention au milieu des humains ne relève ni de l'apprentissage, ni des activités sociales. Aphrodite persuade, elle séduit, en un mot, elle fait perdre la tête ${ }^{42}$. Et l'hymne est une superbe évocation du caractère à la fois effrayant et séduisant de ce sacré, le tremendum et le fascinans ${ }^{43}$ : dans sa relation avec le divin, Anchise est partagé entre l'étonnement, l'admiration, le désir, la crainte et l'effroi ${ }^{44}$.

Il a paru intéressant, dans l'étude d'un sacré spécifique à la déesse, de comparer certains aspects de l'Hymne à Aphrodite avec celui qui chante Déméter. Ces deux poèmes anciens ${ }^{45}$ semblent bien faire partie d'un vaste ensemble théogonique ${ }^{46}$. A plus d'un titre, ils sont normatifs et leur enseignement, fondé sur l'expression mythique d'une action passée, régit des pans importants du sacré.

Aphrodite et Déméter sont des divinités féminines dangereuses dans le cadre de la mise en ordre cosmique. La douleur de la mère menace les équilibres fondamentaux instaurés entre les hommes et les dieux, tandis que

39 Cf. P. SMITH, op. cit., p. 35.

40 Le récit mensonger d'Aphrodite le rappelle encore aux vers 117-121.

41 Hestia est en relation avec la mort animale. Cf. P. SмтTH, op. cit., p. 35-36.

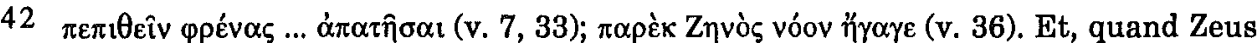
lui insuffle le désir d'Anchise, il applique les mêmes principes qu'Aphrodite :

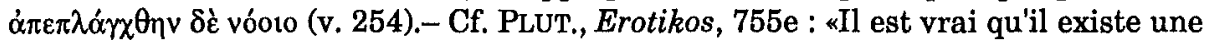
maladie du corps que l'on appelle le mal sacré; il n'y a donc rien d'étonnant que la passion de l'âme qui est la plus furieuse et la plus forte soit dénommée parfois sacrée et divine (íepòv kà̀ $\theta \varepsilon \hat{\imath} o v$ )» (trad. R. Flacelière). Cf. aussi 763a, où Plutarque fait référence à SAPPHO, fr. $31 \mathrm{LP}$.

43 Selon la terminologie de $\mathrm{R}$. Отто, Le sacré. L'élément non-rationnel dans l'idée du divin et sa relation avec le rationnel, trad. d'André Jundt, Paris, Payot, 1949.

44 V. $84,90,91,143,182,187,189,194$.

45 R. JANKo, op. cit., p. 179-180.

46 Cf. J. RUDHARDT, A propos de l'Hymne homérique à Déméter, in op. cit., passim. 
les manifestations extrêmes du pouvoir d'Aphrodite font se poursuivre, sur le plan amoureux, une relation qui, à tous les autres niveaux, s'est établie à distance. Dans les deux cas, c'est au cours d'une ucrise" que les timai des déesses sont définitivement arrêtées, sous l'autorité suprême de Zeus ${ }^{47}$. Et les hommes ont chaque fois reçu une part d'immortalité accordée par ces déesses temporairement humanisées. Sur un plan individuel, Déméter leur a montré une voie d'accès vers un au-delà plein d'espérance. Elle propose une solution divine à la détresse humaine grâce à la révélation des

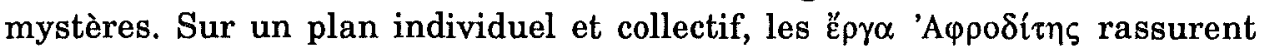
également l'homme qui se perpétue par la descendance.

L'étude du vocabulaire de chaque poème est révélatrice. L'Hymne à Déméter comporte un grand nombre de termes se référant au sacré ${ }^{48}$ tandis

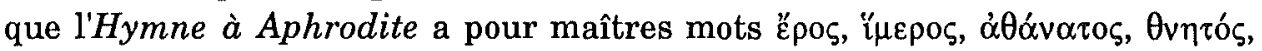

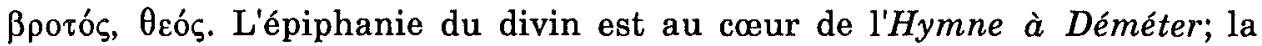
solution que la déesse propose extrait en quelque sorte l'homme de sa condition humaine. Dans l'Hymne à Aphrodite, au contraire, le propos central est le constat d'une altérité définitive entre les dieux et les hommes, et la solution à l'angoisse de la mort, même si elle est présidée par une déesse, reste très humaine ${ }^{49}$. Ainsi chaque hymne exprime dans ses propres termes une forme de sacré spécifique.

\section{Perspectives}

Si l'on se tourne à présent vers l'époque classique, on constate que le Banquet de Platon peut apparaître comme une véritable "théorisation" des propos mythiques de l'Hymne à Aphrodite.

L'amour y est en effet présenté comme un être intermédiaire qui comble le vide entre les hommes et les dieux, étant donné que le divin ne se mêle pas à l'humain ${ }^{50}$. L'amour n'y est ni immortel, ni mortel; sa nature est démonique 51 .

47 Ibid. - Sur la signification symbolique de l'Hymne à Déméter, cf. A. MoTTE, op. cit., p. 194-204.

48 A. MOTTE, op. cit., p. 194; pour une récente comparaison entre l'Hymne à Déméter et l'Iliade, voir A. CHEYNS, La structure du récit dans l'Tliade et l'Hymne homérique à Déméter, in $R B P h, 66$ (1988), p. 32-67.

49 La seule occurrence du mot iepós concerne précisément les offrandes que promet Anchise dans la prière à la déesse; il s'agit donc d'un contexte cultuel (v. 101).

50 Platon, Banquet, 202e-203a.

51 ID., 202e, 203d. Cf. V. PIRENNE-DELFORGE, Éros en Grèce : dieu ou démon ?, in Anges et démons dans les grandes religions. Actes du Colloque de Louvain-la-Neuve et Liege (24-25 nov. 1987), Louvain-la-Neuve (Homo Religiosus, 13), à paraître. 
L'union de l'homme et de la femme est un enfantement et, dans cet acte, réside quelque chose de divin. La fécondité et la procréation confêrent une immortalité au vivant qui est mortel ${ }^{52}$. L'objet de l'amour, c'est de procréer et d'enfanter dans la beauté, car ce que l'être mortel peut avoir de perpétuité et d'immortalité, il l'obtient grâce à la procréation ${ }^{53}$. Donc, l'objet de l'amour est aussi l'immortalité ${ }^{54}$.

Bien qu'il retienne une autre forme d'immortalité en rapport avec sa théorie des Idées, Platon s'attarde longuement, et à plusieurs reprises, au principe de l'immortalité par l'amour. Il est clair que les passages en question ne se réfèrent pas explicitement à l'Hymne à Aphrodite, mais ils véhiculent un même message, chacun dans le langage qui lui est propre. ${ }^{55}$

Pièce galante, poésie de cour, l'Hymne homérique à Aphrodite n'est, semble-t-il, rien de tout cela. Il reflète au contraire une véritable révolution dans la mise en œuvre du cosmos, un pas supplémentaire dans l'accomplissement de la volonté souveraine de Zeus et dans sa définition d'une relation entre les hommes et les dieux. Il s'agit bien d'une poésie sacrée, qui explique peut-être pourquoi les hommes continuent de s'aimer a ujourd'hui...

Université de Liège

Vinciane PIRENNE-DELFORGE aspirant du F.N.R.S.

Place du XX-Août 32, loc. $5 / 19$

B - 4000 LIËGE

52 PLATON, 206c.

53 ID., $206 \mathrm{e}$.

54 ID., 207a. Cf. aussi 207d, 208b, 208d; PLUT., Erotikos, 752a et, surtout, 764d où il rapporte des fables égyptiennes qui rapprochent Aphrodite de la lune, car cet astre participe à la fois de la terre et du ciel, kelle est le lieu où l'élément immortel se mêle au mortel" (trad. R. Flacelière). - L'anthropologie fantastique d'Aristophane, dans le Banquet de Platon, est également intéressante car elle parle d'un temps où l'unité humaine conférait une telle puissance aux hommes qu'ils touchaient au divin. $O r$ c'est précisément cette fusion première que les êtres recherchent dans l'amour.

55 Pour une réflexion approfondie sur le sujet chez Platon, voir l'article du professeur A. Motte dans ce volume. 